\title{
An investigation into design of fair surfaces over irregular domains using data-dependent triangulation
}

\author{
R SHARMA $^{1}$ and O P SHA ${ }^{2}$ \\ Design Laboratory, Department of Ocean Engineering and Naval Architecture, \\ Indian Institute of Technology, Kharagpur 721302, India \\ e-mail: ${ }^{1}$ rajivatri@yahoo.com; ${ }^{2}$ ops@ naval.iitkgp.ernet.in
}

MS received 26 October 2004; revised 12 June 2006

\begin{abstract}
Design of fair surfaces over irregular domains is a fundamental problem in computer-aided geometric design (CAGD), and has applications in engineering sciences (in aircraft, automobile, ship science etc.). In the design of fair surfaces over irregular domains defined over scattered data, it was widely accepted till recently that the classical Delaunay triangulation be used because of its global optimum property. However, in recent times it has been shown that for continuous piecewise linear surfaces, improvements in the quality of fit can be achieved if the triangulation pattern is made dependent upon some topological or geometric property of the data set or is simply data dependent. The fair surface is desired because it ensures smooth and continuous surface planar cuts, and these in turn ensure smooth and easy production of the surface in CAD/CAM, and favourable resistance properties. In this paper, we discuss a method for construction of $C^{1}$ piecewise polynomial parametric fair surfaces which interpolate prescribed $\mathfrak{R}^{3}$ scattered data using spaces of parametric splines defined on $\mathfrak{R}^{3}$ triangulation. We show that our method is more specific to the cases when the projection on a 2- $D$ plane may consist of triangles of zero area, numerically stable and robust, and computationally inexpensive and fast. Numerical examples dealing with surfaces approximated on plates, and on ships have been presented.
\end{abstract}

Keywords. Cubic spline; $C^{1}$ continuous surface; data-dependent triangulation; minimum energy surface; surface fitting.

\section{Introduction}

Surface interpolation to scattered data in $\mathfrak{R}^{3}$ is a problem of general interest cutting across many engineering disciplines. In recent years, there has been great interest generated around this problem, e.g. Franke (1987), Schumaker (1976), Quak \& Schumaker (1990), and references therein. In general the problem of surface interpolation is done by applying the construction of piecewise polynomial parametric surfaces which interpolate prescribed $\mathfrak{R}^{3}$ scattered data using spaces of parametric splines defined on $\mathfrak{R}^{2}$ triangulation. The points in the $\mathfrak{R}^{3}$ are projected on a plane in $\mathfrak{R}^{2}$ and triangulation is defined as a set of stitched non-intersecting 
triangles with the vertices at the points in the point set. The surface $S$ is then defined by the union of triangles in $F$ by interpolating parametric surface of desired degree $d$ and continuity $r$. A unique plane can be defined which passes through the vertices of the triangle and this projection is not difficult. However, if we want to use the same plane for projection over the complete point set, a situation might arise where the triangle in 3D may get transformed into a zero area triangle in $2 \mathrm{D}$ or a triangle in $2 \mathrm{D}$ with poor aspect ratio. Hence, the quality of the fit in this application is dependent on the projection of the $\mathfrak{R}^{3}$ data on the plane in $\mathfrak{R}^{2}$. In general, this is undesirable and may restrict the quality of the surface.

Generally, in surface interpolation a spline of the desired degree and continuity is passed over a triangulation. Since there are many triangulations associated with the scattered data, we have to find the optimum triangulation. As per Schumaker (1987), for scattered data interpolation, classical Delaunay triangulation can be used. However, this is suitable only when the triangulation as a parameter is used for different functions. In more specific cases (i.e. surfaces of mixed continuities), triangulation as a parameter cannot be used for different functions and the choice of triangulation depends upon the function being fitted. In this context Dyn et al $(1989,1990)$ have observed that the quality of the fit can be improved by adjusting the triangulation to the function being fitted. The authors have also discussed that the best way is the minimization of the size of the normal derivative discontinuities across the edge of the triangulation. In the same line, Quak \& Schumaker (1990) have discussed the possibility of applying the construction of piecewise polynomial parametric surfaces which interpolate prescribed $\mathfrak{R}^{3}$ scattered data using spaces of parametric splines defined on $\mathfrak{R}^{2}$ triangulation. The authors use projection of 3D data set on 2D along a plane. However, they have not given any guidelines for the selection of the plane.

The quality of fit in case of surface design not only depends upon the continuities (i.e. geometric $G^{0,1,2}$, or derivational $C^{0,1,2}$ ), but in practical applications in engineering industries also upon the desired fairness properties of the surface. To have a fair surface, many authors suggest incorporation of the principles of physics or optimization techniques in the design process to automatically achieve a desired smooth surface, e.g. Schmidt (1982), Terzopoulos \& Qin (1994), Qin \& Terzopoulos (1996), Fasshauer \& Schumaker (1996), Sarraga (1998), and references therein. In order to achieve the minimum value for an objective function, we have to solve a simultaneous system of linear, or nonlinear equations. The solution of nonlinear equations in general is too expensive to be used in an interactive user environment in engineering sciences, and sometimes no acceptable solution can be computed.

In the present work, we explore the possibility of applying the construction of piecewise polynomial parametric surfaces which interpolate prescribed $\mathfrak{R}^{3}$ scattered data using spaces of parametric splines defined on $\mathfrak{R}^{3}$ triangulation. Since the triangulation is defined in $\mathfrak{R}^{3}$, no projection is required. We have used piecewise cubic $C^{1}$ surfaces and the selection of the triangulation is based on the idea of minimizing the energy of the resulting surface. We have used this objective function (i.e. elastic energy of a thin plate) because it can be approximated numerically, resulting in stable and robust computations, and better computational efficiency.

\section{Surface representation}

The problem addressed in this work is formally stated as here: Given a set of points $P=$ $\left\{\left(x_{i}, y_{i}, z_{i}\right)\right\}_{i=1}^{N}$ in $\mathfrak{R}^{3}$ and a corresponding set of measurements $\left\{G_{i}=f\left(x_{i}, y_{i}, z_{i}\right)\right\}_{i=1}^{N}$ on a function $f$, construct a surface $S$ approximating $f$.

Following Quak \& Schumaker (1990), let $\Delta$ is a triangulation associated with a set $P$ of points as defined previously. In addition, suppose the gradients $\left(f_{x}, f_{y}, f_{z}\right)$ has been estimated 


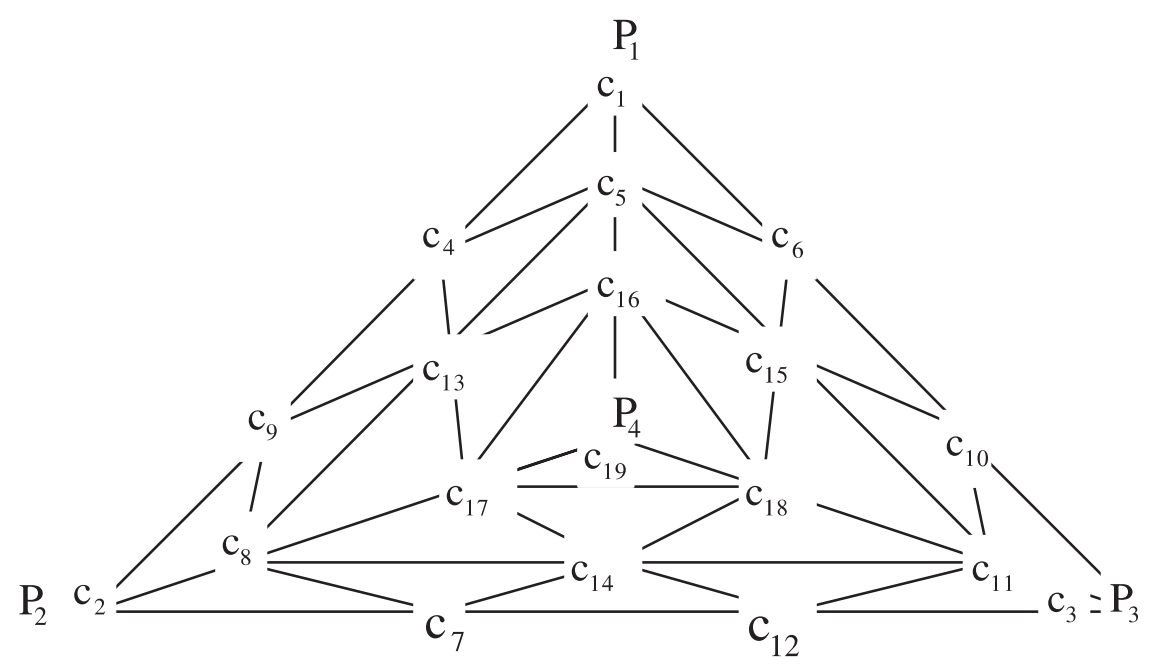

Figure 1. The Clough-Tocher split.

at each of the data points of the dataset (i.e. $\left.\left(x_{i}, y_{i}, z_{i}\right)\right)$, producing the values $\left(G_{i}^{x}, G_{i}^{y}, G_{i}^{z}\right)$, $i=1, \ldots, N$. Let $T$ be a typical triangle in the triangulation $\Delta$, and its vertices be $P_{1}, P_{2}, P_{3}$ and its edges $e_{1}, e_{2}, e_{3}$. By connecting the centroid point $P_{4}=\left(\left(P_{1}+P_{2}+P_{3}\right) / 3\right)$ of the triangle $T$ to each of its vertices, we split $T$ into three sub-triangles as shown in figure 1 . We denote by $T_{1}, T_{2}, T_{3}$ the three subtriangles obtained by connecting the centroid (i.e. $P_{4}$ ) and the vertices $P_{1}, P_{2}, P_{3}$. This split is known as Clough-Tocher split.

As per Lawson (1977) and Renka (1984), there is a unique piecewise cubic function $s_{1}$ in $C^{1}(T)$ which satisfies the interpolation condition as:

$$
s_{T}\left(P_{i}\right)=G_{i}, \quad D_{x} s_{T}\left(P_{i}\right)=G_{i}^{x}, \quad D_{y} s_{T}\left(P_{i}\right)=G_{i}^{y}, \quad D_{z} s_{T}\left(P_{i}\right)=G_{i}^{z},
$$

for $i=1,2,3$ and which has the further property that the normal derivatives along each of the edges $e_{1}, e_{2}, e_{3}$ are linear polynomials rather than quadratics.

Since this property has been combined in the interpolation conditions, it implies that the surface $S$ obtained by stitching together the patches $s_{T}$ is globally $C^{1}$ and satisfies the interpolation conditions as imposed in (1), for all $i=1, \ldots, N$.

Theorem 1. On the triangle T, the spline has the following Bérnstein-Bézier coefficients (figure 1):

$$
\begin{aligned}
& c_{1}=G_{1}, \\
& c_{2}=G_{2}, \\
& c_{3}=G_{3}, \\
& c_{4}=\left[\frac{\left(x_{2}-x_{1}\right) \cdot G_{1}^{x}+\left(y_{2}-y_{1}\right) \cdot G_{1}^{y}+\left(z_{2}-z_{1}\right) \cdot G_{1}^{z}}{3+G_{1}}\right],
\end{aligned}
$$




$$
\begin{aligned}
& c_{5}=\left[\frac{\left(x_{4}-x_{1}\right) \cdot G_{1}^{x}+\left(y_{4}-y_{1}\right) \cdot G_{1}^{y}+\left(z_{4}-z_{1}\right) \cdot G_{1}^{z}}{3+G_{1}}\right], \\
& c_{6}=\left[\frac{\left(x_{3}-x_{1}\right) \cdot G_{1}^{x}+\left(y_{3}-y_{1}\right) \cdot G_{1}^{y}+\left(z_{3}-z_{1}\right) \cdot G_{1}^{z}}{3+G_{1}}\right], \\
& c_{7}=\left[\frac{\left(x_{3}-x_{2}\right) \cdot G_{2}^{x}+\left(y_{3}-y_{2}\right) \cdot G_{2}^{y}+\left(z_{3}-z_{2}\right) \cdot G_{2}^{z}}{3+G_{2}}\right], \\
& c_{8}=\left[\frac{\left(x_{4}-x_{2}\right) \cdot G_{2}^{x}+\left(y_{4}-y_{2}\right) \cdot G_{2}^{y}+\left(z_{4}-z_{2}\right) \cdot G_{2}^{z}}{3+G_{2}}\right], \\
& c_{9}=\left[\frac{\left(x_{1}-x_{2}\right) \cdot G_{2}^{x}+\left(y_{1}-y_{2}\right) \cdot G_{2}^{y}+\left(z_{1}-z_{2}\right) \cdot G_{2}^{z}}{3+G_{2}}\right],
\end{aligned}
$$$$
c_{10}=\left[\frac{\left(x_{1}-x_{3}\right) \cdot G_{3}^{x}+\left(y_{1}-y_{3}\right) \cdot G_{3}^{y}+\left(z_{1}-z_{3}\right) \cdot G_{3}^{z}}{3+G_{3}}\right] \text {, }
$$$$
c_{11}=\left[\frac{\left(x_{4}-x_{3}\right) \cdot G_{3}^{x}+\left(y_{4}-y_{3}\right) \cdot G_{3}^{y}+\left(z_{4}-z_{3}\right) \cdot G_{3}^{z}}{3+G_{3}}\right] \text {, }
$$$$
c_{12}=\left[\frac{\left(x_{2}-x_{3}\right) \cdot G_{3}^{x}+\left(y_{2}-y_{3}\right) \cdot G_{3}^{y}+\left(z_{2}-z_{3}\right) \cdot G_{3}^{z}}{3+G_{3}}\right] \text {, }
$$$$
c_{13}=\left[\frac{\left(c_{5}+c_{8}+\left(\theta_{1}-1\right) \cdot c_{1}\right)+\left(2-3 \theta_{1}\right) \cdot c_{4}+\left(3 \theta_{1}-1\right) \cdot c_{9}-\theta_{1} \cdot c_{2}}{2}\right] \text {, }
$$$$
c_{14}=\left[\frac{\left(c_{8}+c_{11}+\left(\theta_{2}-1\right) \cdot c_{2}\right)+\left(2-3 \theta_{2}\right) \cdot c_{7}+\left(3 \theta_{2}-1\right) \cdot c_{12}-\theta_{2} \cdot c_{3}}{2}\right] \text {, }
$$$$
c_{15}=\left[\frac{\left(c_{11}+c_{5}+\left(\theta_{3}-1\right) \cdot c_{3}\right)+\left(2-3 \theta_{3}\right) \cdot c_{10}+\left(3 \theta_{3}-1\right) \cdot c_{6}-\theta_{3} \cdot c_{1}}{2}\right] \text {, }
$$$$
c_{16}=\left[\frac{c_{15}+c_{5}+c_{13}}{3}\right] \text {, }
$$$$
c_{17}=\left[\frac{c_{13}+c_{8}+c_{14}}{3}\right] \text {, }
$$$$
c_{18}=\left[\frac{c_{14}+c_{11}+c_{15}}{3}\right] \text {, }
$$$$
c_{19}=\left[\frac{c_{18}+c_{16}+c_{17}}{3}\right] \text {, }
$$ 
where,

$$
\begin{aligned}
& \theta_{1}=\left[\frac{\left(x_{4}-x_{1}\right) \cdot\left(x_{2}-x_{1}\right)+\left(y_{4}-y_{1}\right) \cdot\left(y_{2}-y_{1}\right)+\left(z_{4}-z_{1}\right) \cdot\left(z_{2}-z_{1}\right)}{\left(\left(x_{2}-x_{1}\right)^{2}+\left(y_{2}-y_{1}\right)^{2}+\left(z_{2}-z_{1}\right)^{2}\right)}\right], \\
& \theta_{2}=\left[\frac{\left(x_{4}-x_{2}\right) \cdot\left(x_{3}-x_{2}\right)+\left(y_{4}-y_{2}\right) \cdot\left(y_{3}-y_{2}\right)+\left(z_{4}-z_{2}\right) \cdot\left(z_{3}-z_{2}\right)}{\left(\left(x_{3}-x_{2}\right)^{2}+\left(y_{3}-y_{2}\right)^{2}+\left(z_{3}-z_{2}\right)^{2}\right)}\right], \\
& \theta_{3}=\left[\frac{\left(x_{4}-x_{3}\right) \cdot\left(x_{1}-x_{3}\right)+\left(y_{4}-y_{3}\right) \cdot\left(y_{1}-y_{3}\right)+\left(z_{4}-z_{3}\right) \cdot\left(z_{1}-z_{3}\right)}{\left(\left(x_{1}-x_{3}\right)^{2}+\left(y_{1}-y_{3}\right)^{2}+\left(z_{1}-z_{3}\right)^{2}\right)}\right], \\
& x_{4}=\left[\frac{x_{1}+x_{2}+x_{3}}{3}\right], \\
& y_{4}=\left[\frac{y_{1}+y_{2}+y_{3}}{3}\right],
\end{aligned}
$$

and

$$
z_{4}=\left[\frac{z_{1}+z_{2}+z_{3}}{3}\right]
$$

Proof. We consider the polynomial piece $p=s I_{T^{i}}$. Then as per the coefficients shown in figure 1, the Bérnstein-Bézier representation of $p$ is given by,

$$
\begin{aligned}
p(u, v, w)= & c_{19} u^{3}+3 \cdot c_{16} u^{2} v+3 \cdot c_{17} u^{2} w+3 \cdot c_{5} u v^{2}+6 \cdot c_{13} u v w \\
& +3 \cdot c_{8} u w^{2}+c_{1} v^{3}+3 \cdot c_{4} v^{2} w+3 \cdot c_{9} v w^{2}+c_{2} w^{3}
\end{aligned}
$$

where $(u, v, w)$ are the barycentric coordinates of a point $(x, y, z)$ in triangle $T_{1}$ with respect to its vertices $P_{4}, P_{1}, P_{2}$. Now $p$ takes on the value $G_{1}$ at $P_{1}$, if and only if $c_{1}=G_{1}$. Considering the interpolation conditions involving the gradients at $P_{1}$, let $D_{\sigma}$ denote the directional derivative in the direction $\sigma=\left(x_{2}-x_{1}, y_{2}-y_{1}, z_{2}-z_{1}\right)$. Then using the relation between the directional derivative of a polynomial and its Bérnstein-Bézier coefficients (Farin 1980), it follows that

$$
D_{\sigma} p=\left[\frac{\left(c_{4}\left(3-\left(-c_{1}\right)\right)\right)}{\left\{\left(x_{2}-x_{1}\right)^{2}+\left(y_{2}-y_{1}\right)^{2}+\left(z_{2}-z_{1}\right)^{2}\right\}^{1 / 2}}\right] .
$$

In terms of the gradient, the directional derivative can be written as,

$$
D_{\sigma} p=\left[\frac{\left(x_{2}-x_{1}\right) \cdot G_{1}^{x}+\left(y_{2}-y_{1}\right) \cdot G_{1}^{y}+\left(z_{2}-z_{1}\right) \cdot G_{1}^{z}}{\left\{\left(x_{2}-x_{1}\right)^{2}+\left(y_{2}-y_{1}\right)^{2}+\left(z_{2}-z_{1}\right)^{2}\right\}^{1 / 2}}\right] .
$$

At $P_{1}$, equating (2) and (3) we get,

$$
c_{4}=\left[\frac{\left(x_{2}-x_{1}\right) \cdot G_{1}^{x}+\left(y_{2}-y_{1}\right) \cdot G_{1}^{y}+\left(z_{2}-z_{1}\right) \cdot G_{1}^{z}}{3+G_{1}}\right]
$$

which is as given in theorem 1 . Next we consider the derivative at normal to the edge $e_{1}$ and pointing into the triangle, evaluating at a point $(0,1-v, v)$, it can be given by, 
$D_{\sigma} p(0,1-v, v)$

$$
=\left[\begin{array}{c}
3 \cdot\left(\begin{array}{c}
c_{5} \cdot(1-v)^{2}+2 \cdot c_{13} \cdot v(1-v) \\
+c_{8} \cdot v^{2}+c_{1} \cdot(1-v)^{2} \cdot\left(\theta_{1}-1\right)
\end{array}\right) \\
+3 \cdot c_{4} \cdot\left(\begin{array}{c}
-\theta_{1}(1-v)^{2}+2 \cdot\left(\theta_{1}-1\right) \cdot \\
v \cdot(1-v)
\end{array}\right) \\
+3 \cdot c_{9} \cdot\left(\begin{array}{c}
-2 \cdot \theta_{1} \cdot v \cdot(1-v)+\left(\theta_{1}-1\right) \cdot \\
v^{2}-\theta_{1} \cdot v^{2} \cdot c_{2}
\end{array}\right)
\end{array}\right] / d_{P_{4} e_{1}}
$$

where $d_{P_{4} e_{1}}$ is the distance of the point $P_{4}$ from the edge $e_{1}$ and $\theta_{1}$ is as defined in theorem 1 . Since we are discussing the $C^{1}$ continuous surface, the polynomial in (4) consists only of first degree terms. This reduces to a first degree polynomial in $v$, if and only if the second derivative of the polynomial in (4) with respect to $v$ is equal to zero, i.e.,

$$
\begin{aligned}
0= & 2 \cdot c_{3}-4 \cdot c_{13}+2 \cdot c_{8}+2 \cdot c_{1}\left(\theta_{1}-1\right) \\
& +c_{4} \cdot\left(-2 \cdot \theta_{1}-4 \cdot\left(\theta_{1}-1\right)\right)+c_{9} \cdot\left(4 \cdot \theta_{1}+2 \cdot\left(\theta_{1}-1\right)\right)-2 \cdot \theta_{1} \cdot c_{2} .
\end{aligned}
$$

Rearranging the terms in (5), we get,

$$
c_{13}=\left\{\left(c_{5}+c_{8}+\left(\theta_{1}-1\right) \cdot c_{1}\right)+\left(2-3 \theta_{1}\right) \cdot c_{4}+\left(3 \theta_{1}-1\right) \cdot c_{9}-\theta_{1} \cdot c_{2}\right\} / 2
$$

which is as given in theorem 1. Similarly, considering the triangles $T_{2}, T_{3}$ we can get the formulas for the coefficients $c_{6}, c_{7}, c_{10}, c_{11}, c_{12}, c_{14}$ and $c_{13}$.

Considering $C^{1}$ continuity across the interior edges, since the barycentric coordinate of the point $P_{4}$ in terms of $P_{1}, P_{2}$ and $P_{3}$ is $(1 / 3,1 / 3,1 / 3)$, being the centre of gravity of the triangle having vertices at $P_{1}, P_{2}$ and $P_{3}$, let us consider the triangle whose vertices are on $c_{5}, c_{13}$ and $c_{15}$, where $c_{16}$ will be at its centre of gravity being the Clough-Tocher split. This will leads to,

$$
c_{16}=\left[\left(c_{15}+c_{5}+c_{13}\right) / 3\right] .
$$

Similarly,

$$
\begin{aligned}
& c_{17}=\left[\left(c_{13}+c_{8}+c_{14}\right) / 3\right] \text { for a triangle having vertices at } c_{8}, c_{13} \text { and } c_{14}, \text { and, } \\
& c_{18}=\left[\left(c_{14}+c_{11}+c_{15}\right) / 3\right] \text { for a triangle having vertices at } c_{11}, c_{14} \text { and } c_{15} .
\end{aligned}
$$

At last from any one of the $C^{1}$ continuity conditions across an edge we get,

$$
c_{19}=\left[\left(c_{18}+c_{16}+c_{17}\right) / 3\right] \text { for a triangle having vertices at } c_{16}, c_{17} \text { and } c_{18} .
$$

\section{Definition of the energy of the surface}

Following, Quak \& Schumaker (1990), and Fasshauer \& Schumaker (1996), suppose that $\bar{\Delta}=\left\{T_{i}\right\}_{i}^{N}$ is the refined triangulation of $\Delta$ obtained by performing the Clough-Tocher split 
on each of the triangles of $\Delta$. Let $s$ be a piecewise polynomial function over $\bar{\Delta}$. If we think of a surface $S$ as a thin plate (assuming Poisson's ratio is equal to zero), the natural energy measure of the surface is given by,

$$
\partial(s)=\sum_{i=1}^{N} \partial_{i}(s)
$$

where for each triangle $T_{i}$ of $\bar{\Delta}$,

$$
\partial_{T_{i}}(s)=\iint_{T_{i}}\left[\left(u_{x x}\right)^{2}+\left(u_{y y}\right)^{2}+2 \cdot\left(u_{x y}\right)^{2}\right] d x d y, i=1, \ldots \ldots, N .
$$

In (6) for numerical computation, $s$ is represented in classical Bérnstein-Bézier coefficients. Each cubic piece $s_{i}=s I_{T_{i}}$ is described by a vector $c_{i}=\left(c_{i, 1}, c_{i, 2}, \ldots \ldots, c_{i, 10}\right)$. Normally, the authors work with (6) either via nonlinear numerical energy expressions or use numerical integration techniques to evaluate the quadratic energy integral, and both of these are computationally quite expensive. Additionally, because of high computational time it restricts the use of (6) to only few cases where the point set is comparatively small. It is shown by Quak $\&$ Schumaker (1989) that the energy contribution of this piece can be approximated by the quadratic form:

$$
\delta_{T_{i}}(s)=\left\{\{c\}_{i}^{T} \cdot\{E\}_{i}^{(3)} \cdot\{c\}_{i}\right\},
$$

where $\{E\}_{i}^{(3)}$ is a symmetric $10 \times 10$ energy matrix. However, the formulations given in there are for 2D canonical triangles but these can be further extended to 3D canonical triangles (for proof, and details see Sha \& Sharma 1999). Furthermore, it has been shown that (7) is not only numerical stable, but also has low computational time. On a comparative scale, it reduces the computational time by around $40 \%$ (Sha \& Sharma 1999). Moreover, because of low computational time it allows the use of equation (6) to general cases where the point set is comparatively bigger. Owing to symmetries in (7), only 13 entries are sufficient. Since we are considering a rotation-invariant energy expression, let the triangle $T_{i}$ be in the position shown in figure 2. It is to be noted here that the computation for the canonical triangle is for computational efficiency, and any general triangle can be transformed into a canonical triangle with some definitive transformations. These transformations are given in appendix A. We may suppose that the triangle is in a canonical position, as shown in figure 2 , with vertices $P_{1}=(b, c, d), P_{2}=(0,0,0)$, and $P_{3}=(a, 0,0)$.

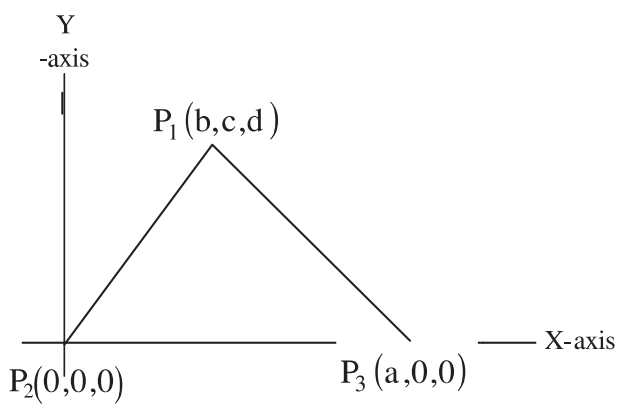

Figure 2. The canonical triangle. 
Now the Barycentric co-ordinates of any point $(x, y, z)$ are given by $(u, v, w)$, where,

$$
\begin{aligned}
u= & {\left[\left(y^{2}+z^{2}\right) /\left(c^{2}+d^{2}\right)\right]^{1 / 2}, } \\
v= & {\left[\left\{(c \cdot z-d \cdot y)^{2}+(d(x-a)+z(a-b))^{2}\right.\right.} \\
& \left.\left.+(y(b-a)+c(a-x))^{2}\right\} / a^{2} \cdot\left(c^{2}+d^{2}\right)\right]^{1 / 2},
\end{aligned}
$$

and $w=(c \cdot z-d \cdot y)^{2}+(d \cdot x+b \cdot z)^{2}+(b \cdot y+c \cdot x)^{2} / a^{2} \cdot\left(c^{2}+d^{2}\right)^{1 / 2}$.

Then the gradients are,

$$
\begin{aligned}
& u=\left(u_{x}, u_{y}, u_{z}\right)=\left(0,\left(\frac{1}{\left(c^{2}+d^{2}\right)^{1 / 2}}\right),-\left(\frac{1}{\left(c^{2}+d^{2}\right)^{1 / 2}}\right)\right), \\
& v=\left(v_{x}, v_{y}, v_{z}\right)=\left(-\frac{1}{a}, \frac{1}{a}\left[\frac{(b-a)^{2}+d^{2}}{c^{2}+d^{2}}\right]^{1 / 2}, \frac{1}{a}\left[\frac{c^{2}+(a-b)^{2}}{c^{2}+d^{2}}\right]^{1 / 2}\right),
\end{aligned}
$$

and $w=\left(w_{x}, w_{y}, w_{z}\right)=\left(\frac{1}{a},-\frac{1}{a}\left[\frac{b^{2}+d^{2}}{c^{2}+d^{2}}\right]^{1 / 2}, \frac{1}{a}\left[\frac{b^{2}+d^{2}}{c^{2}+d^{2}}\right]^{1 / 2}\right)$.

The 13 essential entries (Sha \& Sharma 1999; Quak \& Schumaker 1989; Quak \& Schumaker $1990)$ of $G S=5\left(E_{1}^{(3)} / 9 a . c\right)$ are then given by,

$$
\begin{aligned}
G S_{1,1}= & (U \cdot U)^{2}, \\
G S_{1,2}= & 2 \cdot(U \cdot U)(U \cdot V)+0.5(U \cdot U)^{2}, \\
G S_{1,4}= & (U \cdot V)^{2}+(U \cdot U)(U \cdot V), \\
G S_{1,5}= & 2 \cdot(U \cdot V)(U \cdot W)+(U \cdot U)(U \cdot W)+(U \cdot U)^{2}, \\
G S_{1,7}= & 0.5(U \cdot V)^{2}, \\
G S_{1,8}= & (U \cdot V)(U \cdot W)+0.5(U \cdot V)^{2}, \\
G S_{2,2}= & 2 \cdot(U \cdot U)(V \cdot V)+2(U \cdot V)^{2}+2(U \cdot U)(U \cdot V)+(U \cdot U)^{2}, \\
G S_{2,3}= & 2 \cdot(U \cdot U)(V \cdot W)+2(U \cdot V)(U \cdot W)+(U \cdot U)(U \cdot W), \\
& +(U \cdot U)(U \cdot V)+0.5(U \cdot U)^{2} \\
G S_{2,4}= & 2 \cdot(V \cdot V)(V \cdot U)+(U \cdot U)(V \cdot V)+1.5(U \cdot U)^{2} \\
& +2(U \cdot U)(U \cdot V) \\
G S_{2,5}= & 2 \cdot(V \cdot V)(U \cdot W)+2(U \cdot V)(V \cdot W)+(U \cdot U)(V \cdot W), \\
& +2(U \cdot V)(U \cdot W)+(U \cdot U)(V \cdot V)+(U \cdot V)^{2}+2(U \cdot U)(U \cdot W) \\
& +(U \cdot U)(U \cdot V)
\end{aligned}
$$




$$
\begin{aligned}
G S_{2,6}= & 2 \cdot(U \cdot W)(V \cdot W)+0.5(U \cdot W)^{2}+(U \cdot U)(V \cdot W) \\
& +(U \cdot V)(U \cdot W)+(U \cdot U)(U \cdot W) \\
G S_{2,9}= & (U \cdot W)(V \cdot W)+(V \cdot V)(U \cdot W) \\
& +(U \cdot V)(V \cdot W)+(U \cdot W)^{2}+(U \cdot V)(U \cdot W), \\
G S_{5,5}= & 2 \cdot\left[(V \cdot V)(W \cdot W)+(V \cdot W)^{2}+(W \cdot W)(U \cdot W)\right. \\
& +(U \cdot W)(V \cdot W)+(V \cdot V)(U \cdot W)+(U \cdot V)(V \cdot W) \\
& +(U \cdot U)(W \cdot W)+(U \cdot W)^{2}+(U \cdot U)(V \cdot W) \\
& \left.+(U \cdot V)(U \cdot W)+(U \cdot U)(V \cdot U)+(U \cdot V)^{2}\right],
\end{aligned}
$$

where,

$$
U=u_{x} i+u_{y} j+u_{z} k, \quad V=v_{x} i+v_{y} j+v_{z} k, \text { and } W=w_{x} i+w_{y} j+w_{z} k
$$

and since the matrix is symmetrical, $G S_{i j}=G S_{j i}$. In the above expressions, $U, V, W$ are vectors and the expressions $U \cdot U, U \cdot V$ etc., represent their dot products. Thus, for example,

$$
V . V=\left(v_{x}^{2}+v_{y}^{2}, v_{z}^{2}\right)=\left(\begin{array}{l}
(1 / a)^{2}+\left(\left\{(b-a)^{2}+d^{2}\right\} /\left\{a^{2} \cdot\left(c^{2}+d^{2}\right)\right\}\right)+ \\
\left(\left\{c^{2}+(a-b)^{2}\right\} /\left\{a^{2} \cdot\left(c^{2}+d^{2}\right)\right\}\right)
\end{array}\right) .
$$

\section{Numerical examples}

We present two algorithms for producing data-dependent triangulation based upon idea of minimizing the energy of the corresponding piecewise $C^{1}$ surface. We have considered the quadrilaterals, which are formed by the two triangles sharing an interior edge. The modification in the triangulation is done by a swap test and is completely local in nature. Hence, to compute the energies in the swap test only the four vertices of the quadrilateral are used.

\section{Algorithm 1}

(1) Define an initial triangulation $F$.

(2) Split the initial triangulation using Clough-Tocher split.

(3) Compute the energies of the each quadrilateral in the triangulation as defined in step 2 .

(4) Arrange the energies into a vector $\partial_{1} \geq \ldots \ldots \ldots \partial_{p}$.

(5) For $i=1, \ldots, p$,

if a split (Clough-Tocher) in quadrilateral $i$ is possible with a reduction in energy then split the triangle and return to step 2.

Otherwise stop.

(6) Stop.

\section{Algorithm 2}

In any algorithm of Delaunay triangulation replace the angle test with the energy swap test as defined in algorithm 1 .

Both the algorithms 1 and 2 have been implemented in C++ on a on a Silicon Graphics ${ }^{T M *}$ Origin $^{T M *} 200$ workstation. The surface views are presented in AutoCAD ${ }^{T M * *} \mathrm{R} 2000$. To illustrate the two algorithms we consider the following problems. 
(a)

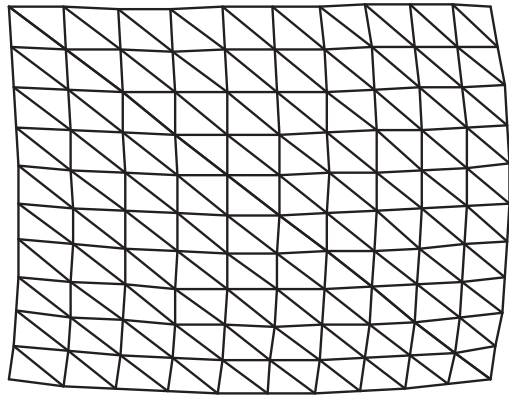

(b)

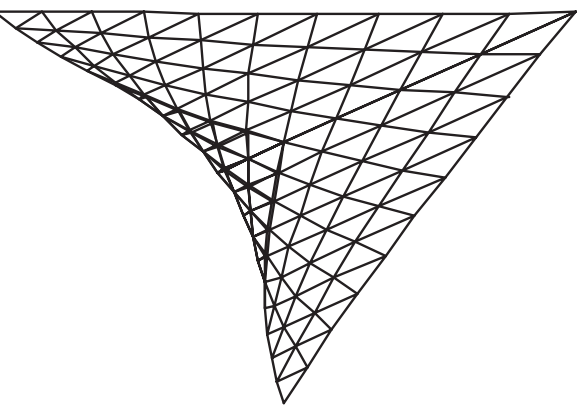

Figure 3. A rectangular plate with one corner raised, $C^{1}$ continuous surface. (a) Top view; (b) isometric view.

Problem 1. This is a simple case where we have used two patches to model a rectangular plate in which one corner has been raised. Let $P_{1}=(0,0,1), P_{2}=(1,1,1), P_{3}=(0,1,0)$ and $P_{4}=$ $(1,0,0)$. In the initial triangulation, to start with, we have divided the rectangle along the line about which the curvature is changing. Suppose the indices of the vertices of the initial triangulation $F$ are given by $(1,2,3)$ and $(3,2,4)$.

Discussion: Case 1 - In this case, figure 3a shows the front view in which the connectivity and triangulation pattern are clear. Here, we have fitted the $C^{1}$ continuous surface over Delaunay triangulation. This can be described as the basic surface and is used to study the behaviour in other cases. Figure $3 \mathrm{~b}$ shows the isometric view. This surface has energy of 53.7 and an error (in discrete maximum norm) of 0.067. In figure 3a, the curvature along the edges is clear. Edges with greater curvature imply higher inherent energy in the surface, and hence less fairness. Since here the modelling is done without any minimization, this is obvious. Figure $3 b$ shows the NW isometric view in which the curved sides, the twist in surface and the higher curvatures are visible. The surface is non-flat, but is $C^{1}$ continuous, so the intersection curves are nonlinear but continuous. Furthermore since it is free from undesirable oscillations, it is clear from figures $3 \mathrm{a}$ and $\mathrm{b}$ that the surface is a fair surface.

Case 2. Here $C^{1}$ continuity has been imposed across the patches and a spline has been passed over triangulation, based upon gradient. Figure $4 \mathrm{~b}$ shows the isometric view for a $C^{1}$ continuous surface. It is clear that the quality of the surface has been improved. All plate corners are not distorted and two of the edges are not curved. A closer look at the surface reveals that the stress concentration is greater in this surface as compared to figure 3 . This surface has an energy of 23.7 and an error (in discrete maximum norm) of 0.079 . The surface has low inherent energy, as can be seen from low curvatures, which is evident from the fact that the modelling has been done by minimization of bending energy. However, the pattern of triangulation is similar in figure 3 , which might be because of the reason that initially the triangulation has been defined over too many points and that may lead to a global optimal solution. If the initial triangulation is close to global optimal, there is less scope of refinement. Again this requires further investigation. An edge with low curvature implies low inherent energy in the surface, and hence high fairness. Figure $4 \mathrm{~b}$ shows the isometric view in which the less curved sides, twist in surface and the lower curvatures are visible. The surface is non-flat but is $C^{1}$ continuous, so the intersection curves are nonlinear but continuous; and the 
(a)

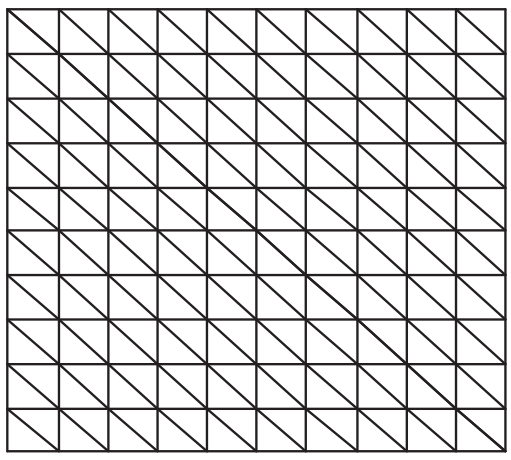

(b)

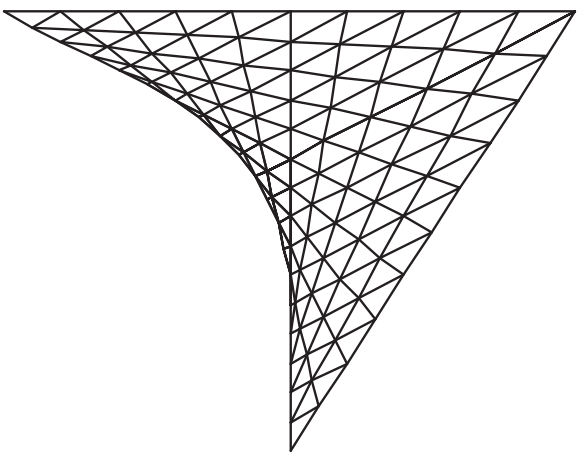

Figure 4. A rectangular plate, $C^{1}$ continuous surface over data dependent triangulation. (a) Top view; (b) isometric view.

nonlinearity is less as compared to case 1 . Furthermore, it is clear from figures $4 a$ and $b$ that the surface is a fair surface.

Problem 2. This is a case of a patch taken on the forward part of a hard chine-fishing vessel. It has been modelled with $C^{1}$ continuity across the interior edges. Let $P_{1}=(13 \cdot 0,3 \cdot 275,3 \cdot 53)$, $P_{2}=(13 \cdot 0,2 \cdot 990,0 \cdot 775), P_{3}=(18 \cdot 0,2 \cdot 672,3 \cdot 855), P_{4}=(18 \cdot 0,2 \cdot 226,1 \cdot 512), P_{5}=$ $(21.5,1.0904,4.202), P_{6}=(21.5,0.5322,2.58), P_{7}=(22.306,0.4655,4.2797), P_{8}=$ $(22 \cdot 306,0.0,2.90)$ and $P_{9}=(22 \cdot 8,0 \cdot 0,4 \cdot 35)$. In the initial triangulation, to start with we have divided the rectangle along the line about which the curvature is changing. Suppose the indices of the vertices of the initial triangulation $F$ are given by $(1,2,3),(2,3,4),(3,4,5)$, $(4,5,6),(5,6,7),(6,7,8)$, and $(7,8,9)$.

Discussion: Figure 5a shows the front view in which the projected triangulation and modelling pattern are clear. Figure 5b shows a typical view of the fishing vessel, where the $C^{1}$ continuous surface is clear.

This surface has an energy of 33.7 and an error (in discrete maximum norm) of 0.076 . In figure 5a, no curvature along the edges is visible. An edge with no visible curvature implies very low inherent energy in the surface, and hence good fairness. Since here the modelling is
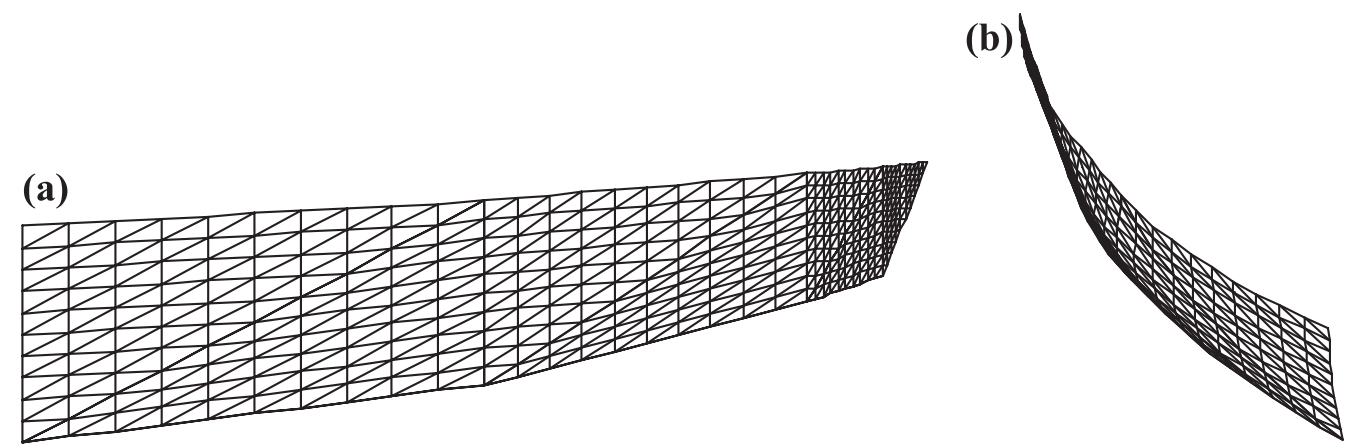

Figure 5. Surface defined over data dependent triangulation in problem 2; (a) top view (b) isometric view 
done with minimization, this implies that the designed fair surface has good quality and it can be used in industry. Figure $5 \mathrm{~b}$ shows the isometric view in which also there is no visible twist, or curvature along the edges. The surface is non-flat but is $C^{1}$ continuous, so the intersection curves will be nonlinear but continuous. Furthermore, it is clear from figure $5 \mathrm{a}$ and $\mathrm{b}$, that the surface is a fair surface.

\section{Conclusions}

As an extension of the work of Quak \& Schumaker (1990), the present work has discussed a general problem, and explored the possibility of applying the construction of piecewise polynomial parametric surfaces, which interpolate prescribed $\Re^{3}$ scattered data using spaces of parametric splines defined on $\mathfrak{R}^{3}$ triangulation to design a fair surface over scattered data in $\mathfrak{R}^{3}$. Since in this work the triangulation is defined in $\mathfrak{R}^{3}$, no projection is required, making it more specific to the cases where the data points have a tendency to converge/collapse. We have shown via theoretical analysis and implementation with two simple problems that the developed algorithm is stable and computationally efficient. A complete complexity analysis of the algorithm with detailed comparative study with other similar techniques has not been attempted. We have used piecewise cubic $C^{1}$ surfaces and the selection of the triangulation is based on the idea of minimizing the energy of the resulting surface, hence the designed fair surface has a minimum physically meaningful property. Initial geometry definition as triangulation can be used to have local control in various applications in the areas of CAGD. However, further improvements of the present surface generation over data-dependent techniques in the application areas of automation, anisotropic generation, adaptation and integration with CFD or D-NURBS solution processes may be needed for development of efficient analytical tools. Our future work will be in this direction, and currently this is under investigation.

This research was partially supported by the Defence Research and Development Organization Govt. of India. The authors thank Prof R P Gokarn for his assistance and comments.

\section{List of symbols}

$C^{r} \quad r$ th order continuity;

$F \quad$ initial triangulation;

$G \quad$ a gradient function;

$N \quad$ number of triangles;

$P \quad$ point set as $\left\{\left(x_{i}, y_{i}, z_{i}\right)\right\}_{i=1}^{N}$;

$S_{d}^{r}(\Delta) \quad$ a spline of degree $d$ and continuity $r$ defined over $\Delta$;

$T_{F} \quad$ transformation matrix;

$\delta_{T_{i}}(s) \quad$ energy expression for $T^{i}$ triangle;

$\Delta \quad$ triangulation defined over $P$;

\section{Appendix A}

Let the coordinates of a general triangle be $\left(x_{1}, y_{1}, z_{1}\right),\left(x_{2}, y_{2}, z_{2}\right)$ and $\left(x_{3}, y_{3}, z_{3}\right)$ as shown in figure A1. 


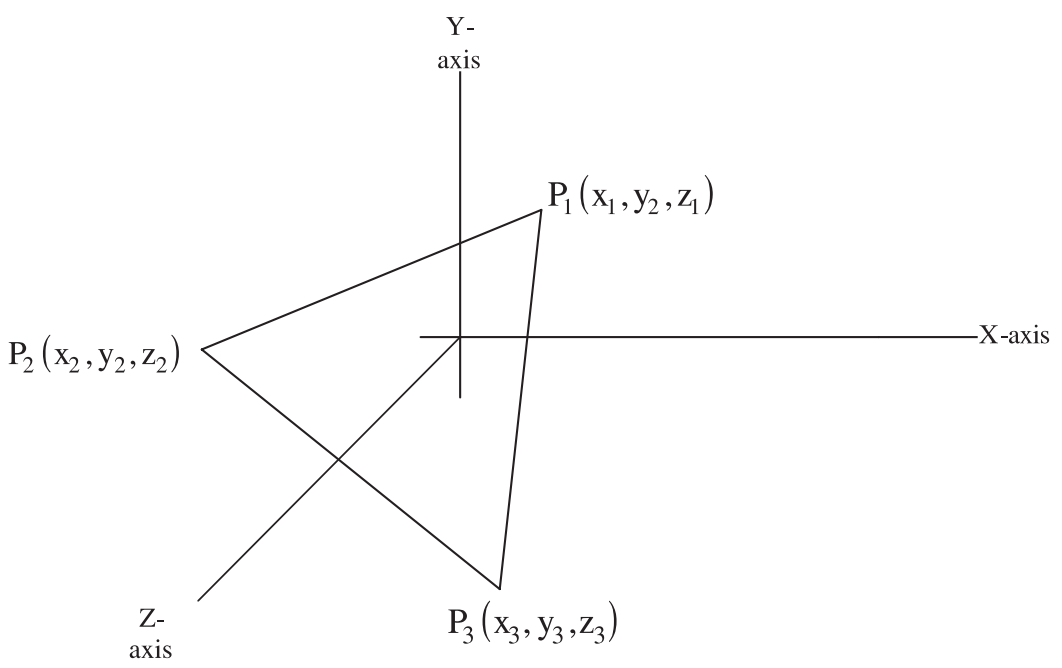

Figure A1. A general triangle.

This can be transformed to rotation invariant canonical position triangle as shown in figure 2 by performing the following transformations.

I. Transferring the origin to any of the vertices of the triangle. The new coordinates are given by

$$
\left\{\begin{array}{llll}
x^{*} & y^{*} & z^{*} & 1
\end{array}\right\}=\left\{\begin{array}{llll}
x & y & z & 1
\end{array}\right\} \cdot\left\{T_{F 1}\right\},
$$

where $\left\{T_{F 1}\right\}$ is the first transformation matrix. Suppose we transfer the origin to $\left(x_{1}, y_{1}, z_{1}\right)$, then the $\left\{T_{F 1}\right\}$ can be written as

$$
\left\{T_{F 1}\right\}=\left\{\begin{array}{llll}
1 & 0 & 0 & 0 \\
0 & 1 & 0 & 0 \\
0 & 0 & 1 & 0 \\
-x_{1} & -y_{1} & -z_{1} & 1
\end{array}\right\} .
$$

II. Rotating in the $X-Y$ plane by angle $\theta$ (clockwise about the $Z$-axis). Since the rotation is in the $X-Y$ plane $z$ remains constant, and

$$
\begin{aligned}
\cos \theta & =\left(x_{2}-x_{1}\right) /\left[\left(x_{2}-x_{1}\right)^{2}+\left(y_{2}-y_{1}\right)^{2}\right]^{1 / 2}, \\
\sin \theta & =\left(y_{2}-y_{1}\right) /\left[\left(x_{2}-x_{1}\right)^{2}+\left(y_{2}-y_{1}\right)^{2}\right]^{1 / 2} .
\end{aligned}
$$

The second transformation matrix can be written as

$$
\left\{T_{F 2}\right\}=\left\{\begin{array}{cccc}
\frac{\left(x_{2}-x_{1}\right)}{\left[\left(x_{2}-x_{1}\right)^{2}+\left(y_{2}-y_{1}\right)^{2}\right]^{1 / 2}} & \frac{-\left(y_{2}-y_{1}\right)}{\left[\left(x_{2}-x_{1}\right)^{2}+\left(y_{2}-y_{1}\right)^{2}\right]^{1 / 2}} & 0 & 0 \\
\frac{\left(y_{2}-y_{1}\right)}{\left[\left(x_{2}-x_{1}\right)^{2}+\left(y_{2}-y_{1}\right)^{2}\right]^{1 / 2}} & \frac{\left(x_{2}-x_{1}\right)}{\left[\left(x_{2}-x_{1}\right)^{2}+\left(y_{2}-y_{1}\right)^{2}\right]^{1 / 2}} & 0 & 0 \\
0 & 0 & 1 & 0 \\
0 & 0 & 0 & 1
\end{array}\right\} .
$$


III. Rotating in the $X-Z$ plane by angle $\phi$ (clockwise about $Y$-axis). Since the rotation is in $X-Z$ plane $y$ will remains constant, and

$$
\begin{aligned}
\cos \phi & =\frac{\left[\left(x_{2}-x_{1}\right)^{2}+\left(y_{2}-y_{1}\right)^{2}\right]^{1 / 2}}{\left[\left(x_{2}-x_{1}\right)^{2}+\left(y_{2}-y_{1}\right)^{2}+\left(z_{2}-z_{1}\right)^{2}\right]^{1 / 2}}, \\
\sin \phi & =\frac{\left(z_{2}-z_{1}\right)}{\left[\left(x_{2}-x_{1}\right)^{2}+\left(y_{2}-y_{1}\right)^{2}+\left(z_{2}-z_{1}\right)^{2}\right]^{1 / 2}} .
\end{aligned}
$$

The third transformation matrix can be written as,

$$
\left\{T_{F 3}\right\}=\left\{\begin{array}{cccc}
\frac{\left[\left(x_{2}-x_{1}\right)^{2}+\left(y_{2}-y_{1}\right)^{2}\right]^{1 / 2}}{\left[\left(x_{2}-x_{1}\right)^{2}+\left(y_{2}-y_{1}\right)^{2}+\left(z_{2}-z_{1}\right)\right]^{1 / 2}} & \frac{-\left(z_{2}-z_{1}\right)}{\left[\left(x_{2}-x_{1}\right)^{2}+\left(y_{2}-y_{1}\right)^{2}+\left(z_{2}-z_{1}\right)\right]^{1 / 2}} & 0 & 0 \\
0 & 0 & 0 & 0 \\
\frac{\left(z_{2}-z_{1}\right)}{\left[\left(x_{2}-x_{1}\right)^{2}+\left(y_{2}-y_{1}\right)^{2}+\left(z_{2}-z_{1}\right)\right]^{1 / 2}} & \frac{\left[\left(x_{2}-x_{1}\right)^{2}+\left(y_{2}-y_{1}\right)^{2}\right]^{1 / 2}}{\left[\left(x_{2}-x_{1}\right)^{2}+\left(y_{2}-y_{1}\right)^{2}+\left(z_{2}-z_{1}\right)\right]^{1 / 2}} & 1 & 0 \\
0 & 0 & 0 & 1
\end{array}\right\} .
$$

Finally the transformation matrix is,

$$
\begin{aligned}
\left\{T_{F}\right\} & =\left\{T_{F 1}\right\} \cdot\left\{T_{F 2}\right\} \cdot\left\{T_{F 3}\right\} \text { and }\left\{\begin{array}{llll}
x^{*} & y^{*} & z^{*} & 1
\end{array}\right\} \\
& =\left\{\begin{array}{llll}
x & y & z & 1
\end{array}\right\} \cdot\left\{T_{F}\right\} .
\end{aligned}
$$

\section{References}

Dyn N, Levin D, Rippa S 1990 Data dependent triangulations for piecewise linear interpolation. IMA J. Numer. Anal. 10: 137-154

Dyn N, Levin D, Rippa S 1989 Algorithms for the construction of data dependent triangulation. In Algorithms for approximation II (eds) M G Cox, J C Mason (Oxford: Clarendon) pp 21-31

Farin G 1980 Bézier polynomials over triangles and the construction of piecewise $C^{r}$ polynomials. Technical Report TR-91, Brunel University, Uxbridge, UK, pp 1-31

Fasshauer E G, Schumaker L L 1996 Minimal energy surfaces using parametric splines. Comput. Aided Geom. Design 13: 45-79

Franke R 1987 Recent advances in the approximation of surfaces from scattered data. In Topics in multivariate approximation (eds) C K Chui, L L Schumaker, F Uterras (New York: Academic Press) 79-98

Qin H, Terzopoulos D 1996 D-NURBS: A physics-based geometric design framework. IEEE Trans. Visual. Comput. Graphics 2: 85-96

Quak E, Schumaker L L 1990 Cubic spline fitting using data dependent triangulations. Comput. Aided Geom. Design 7: 293-301

Quak E, Schumaker L L 1989 Calculation of the energy of a piecewise polynomial surfaces. In Algorithms for approximation II (ed.) M G Cox, J C Mason (Oxford: Clarendon) pp 23-31

Lawson C 1977 Software for $C^{1}$ surface interpolation. In Mathematical software III (ed.) J R Rice (New York: Academic Press) pp 161-194

Renka R J 1984 Triangulation and interpolation at arbitrarily distributed points in the plane algorithm 624. ACM Trans. Math. Software 10: 440-442

Sarraga R F 1998 Recent methods for surface shape optimization. Comput. Aided Geom. Design 15: $417-436$ 
Schmidt R 1982 Eine Methode zur Konstruktion von $C^{1}$-Flächen zur Interpolation unregelmässig vereilter Daten. In Multivariate approximation theory II (eds) W Schempp, K Zeller (Basel: Birlthäuser) pp 343-361

Schumaker L L 1976 Fitting surfaces to scattered data. In Approximation theory II (eds) G G Lorentz, C K Chui, L L Schumaker (New York: Academic Press) pp 203-268

Sha O P, Sharma R 1999 Hull surface modelling with triangular patches Design Laboratory Memorandum 17 - 1999, Department of Ocean Engineering and Naval Architecture, Indian Institute of Technology, Kharagpur, pp 1-56

Terzopoulos D, Qin H 1994 Dynamic NURBS with geometric constraints for interactive sculpting. ACM Trans. Graphics 13: 103-136 